\title{
Automaticity in motor sequence learning does not impair response inhibition
}

\author{
Jessica R. Cohen and Russell A. Poldrack \\ University of California, Los Angeles, California
}

\begin{abstract}
We examined the relationship between automaticity and response inhibition in the serial reaction time (SRT) task to test the common assertion that automatic behavior is ballistic. Participants trained for $3 \mathrm{~h}$ on the SRT, using blocks of a second-order conditional sequence interleaved with random blocks. Automaticity was measured using a concurrent secondary letter-counting task. Response inhibition was measured using a stop-signal task. RTs decreased with training, with a greater decrease for sequenced versus random blocks. Training correlated with a decreased RT cost to performing the secondary task concurrently with the SRT, indicating the development of automaticity. Crucially, there was no change in the ability to inhibit responses at the end of training, even in individuals who showed no dual-task interference. These results demonstrate that the ability to inhibit a motor response does not decrease with automaticity, suggesting that some aspects of automatic behavior are not ballistic.
\end{abstract}

Extensive practice on cognitive or motor tasks is associated with a decreased need for attention and cognitive control, referred to as automaticity. Experimentally, automaticity can be indexed as the cost of performing a secondary task; whereas novel performance is slowed by a concurrent secondary task, practiced performance can proceed without dual-task costs (Logan, 1979; Posner \& Snyder, 1975). A fundamental but heretofore unanswered question concerns the relationship between automaticity and control: Is automatic behavior less controllable?

Schneider and Shiffrin (1977) asserted that "once learned, an automatic process is difficult to suppress, to modify, or to ignore" (p. 2). This is because stimulusresponse mappings become stored relatively permanently; activating a certain stimulus will, therefore, automatically initiate its associated response, a process that simultaneously improves performance and impairs the ability to make alternate responses. In support of their theory, Schneider and Shiffrin found that consistent stimulus-response mapping resulted in learning and automaticity accompanied by difficulty in overriding learned responses, whereas inconsistent stimulusresponse mapping did not produce automaticity or impairment in overriding responses (Shiffrin \& Schneider, 1977). They concluded that the lack of attentional and cognitive resources allotted to automatic behaviors made inhibition of the automatic response more difficult than that of the controlled task.

In contrast to these claims for the ballistic nature of automatic behavior, a study of expert typists by Logan (1982) suggested that automatic behavior might be highly controllable. Logan (1982) operationalized control in terms of response inhibition using the stop-signal paradigm, which has been used to explore executive control in a variety of contexts (see reviews by Lijffijt, Kenemans, Verbaten, \& van Engeland, 2005; Logan, 1994). In this paradigm, participants receive a "stop signal," which may be a tone or a visual signal, on a small proportion of trials while performing a primary task that is often, but not always, a two-choice reaction time (RT) task. Other primary tasks include continuous tasks such as typing, speaking, or arm movements (Logan, 1994). The stop signal tells participants to suppress their current response. Under the assumption of a race between go and stop processes (cf. Band, van der Molen, \& Logan, 2003; Logan \& Cowan, 1984), stop-signal RT is a measure of the time it takes for participants to inhibit their response.

Typing expertise has been associated with a number of variables, such as increased eye-hand span (the number of letters "previewed" while typing), reduced variability in the speed of typing certain letters, and increased speed at initiating words (Salthouse, 1984). Each of these changes intuitively seems to make stopping typing more difficult. For example, eye-hand span reflects a greater ability to prepare to type more letters in advance. Nevertheless, Logan (1982) found that expert typists could successfully and quickly interrupt typing midword when hearing a stop signal; this indicates that the presumed automatic behavior of typing was not ballistic at the level of the word. Moreover, additional analyses demonstrated that skill level, as indexed by words typed per minute, was not related to stopping latency (Logan, 1983).

The results from Logan (1982) regarding expert typists are interesting, especially considering that the participants

J. R. Cohen, jrcohen@psych.ucla.edu 
were professional and highly skilled, and that their typing behavior was, therefore, quite automatic. However, the results are limited for two reasons. First, experts were not directly compared with novices. Second, it is possible that the participants, in addition to being very experienced in typing, were also very experienced in stopping typing due to sudden auditory interruptions (i.e., a phone ringing). In order to more systematically determine the relation between automaticity and response inhibition, we examined the effects of training on stop-signal inhibition in a serial reaction time (SRT) task (Nissen \& Bullemer, 1987) in participants who began as novices in both domains. In the SRT, stimuli appear successively in one of four locations in blocks of either random or sequenced order. With training, participants become faster on sequenced than on random blocks (Nissen \& Bullemer, 1987). The sequence is intricate enough for many participants to remain unaware of it; the task thus measures implicit sequence learning (Willingham, Nissen, \& Bullemer, 1989; but see Fletcher et al., 2005; Perruchet \& Amorim, 1992). A subset of participants often develops at least partial awareness of the sequence (Willingham et al., 1989). Early neuroimaging studies suggested that explicit and implicit learning might engage different neural systems (e.g., Hazeltine, Grafton, \& Ivry, 1997), but recent neuroimaging studies found that, even with explicit knowledge, the neural circuitry involved in implicit learning was active during the SRT (Aizenstein et al., 2004; Willingham, Salidis, \& Gabrieli, 2002). Therefore, the SRT can become automatic through implicit skill learning, even with explicit knowledge. We found (Poldrack et al., 2005) that training on the SRT leads to the development of automaticity within $3 \mathrm{~h}$, as defined by significantly less RT slowing on the SRT due to a secondary task (though see Pashler, 1998, for a criticism of this definition of automaticity).

Combining the SRT with the stop-signal paradigm provides a controlled manner by which to study the relationship between automatic behavior and response inhibition. By measuring stopping latency at different points during training of the SRT, it is possible to determine whether the level of automaticity of a task has an influence on one's ability to inhibit a response. On the basis of Logan's (1982) results with expert typists, we predicted that dual-task interference would decrease with training on the SRT, whereas stop-signal RT would be unaffected by training.

\section{METHOD}

\section{Participants}

Forty-seven right-handed participants (mean age, 21.1 years), 28 women and 19 men, participated for course credit or payment. All participants provided informed consent. Potential participants were excluded if they were taking psychoactive medication or had a history of neurologic illness.

\section{Data Acquisition}

The MATLAB (The MathWorks, Inc., Natick, MA) Psychophysics Toolbox (Brainard, 1997) was used to present stimuli to participants and to record responses on an Apple eMac.

\section{Task Design}

SRT. For SRT (Nissen \& Bullemer, 1987) training, participants viewed a target ("X") on a computer, which appeared in one of four positions across the monitor. Participants were instructed to push the corresponding button on a response box with the right hand as quickly and as accurately as possible. It was emphasized that both speed and accuracy were equally important. The target appeared for $1,000 \mathrm{msec}$, with a 250 -msec interval between trials. There were 40 blocks of 60 trials in each session. The blocks alternated between "sequenced" and "random" conditions, counterbalanced across participants. This alternating design was chosen to minimize explicit knowledge and to control for general practice effects (Poldrack et al., 2005). Unbeknownst to participants, the stimulus locations followed a second-order conditional sequence of 12 items (1-2-3-1-4-3-4-2-1-3-2-4) during sequenced blocks. The sequence was repeated five times in each block and began at different, randomly selected points in different blocks. Every location and every first-order transition occurred equally often. During random blocks, stimulus location followed a pseudorandom order, constrained so that no target location appeared twice in a row and each target location occurred equally often as in sequenced blocks. Sixty pseudorandom trials were initially presented to all participants for practice. The 40 task blocks were administered under singletask, dual-task, or stop-signal conditions. During the first 4 and last 4 blocks, the SRT was combined with the stop-signal paradigm (stop-signal blocks). Immediately following or preceding the stopsignal blocks were 2 single-task blocks in which only the SRT was performed. Immediately following or preceding those single-task blocks were 4 dual-task blocks in which the SRT was performed with a concurrent letter-counting task. The 20 blocks in the middle were single-task blocks. This design ensured that control over the SRT was assessed both as early in training and as late in training as possible, and that dual-task interference (viewed as an index of automaticity) could also be determined both relatively early and relatively late in training for each session; see Figure 1.

Dual task. The secondary task was a letter-counting task, in which a spoken letter ("A" or "B") was presented aurally each time a stimulus appeared. The letters were presented in random order, in approximately equal proportions. Participants counted the number

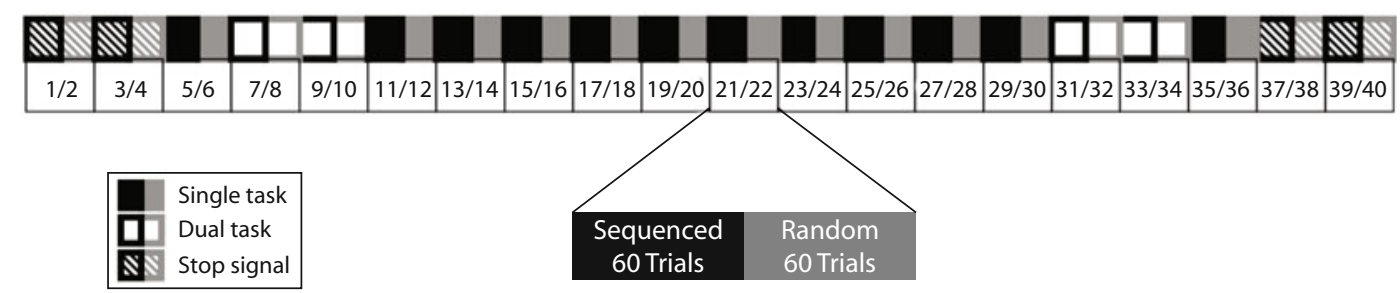

Figure 1. Task design. Participants received 40 blocks of alternating sequenced and random trials in single-task, dual-task, or stop-signal conditions. Sequenced/random order was counterbalanced across participants. 
of Bs while performing the SRT on both sequenced and random blocks; they were told that both tasks were equally important. At the end of each block, they recorded the number of Bs they had heard.

Stop signal. Stop-signal blocks (Logan, 1994) consisted of the SRT with auditory stop signals occurring on $33 \%$ of the trials ("stop" trials) in each block. Stop trials occurred for both sequenced and random blocks of the SRT. Participants attempted to withhold their response on stop trials. It was emphasized that both responding and inhibiting responses were equally important. The stop signal was presented at varying delays (the stop-signal delay, or SSD) after the onset of the SRT stimulus in order to accurately estimate each participant's stop-signal RT (SSRT). The SSD was systematically varied using two independent staircase functions (beginning at $150 \mathrm{msec}$ ) on alternating trials in each block. SSD increased by $50 \mathrm{msec}$ if participants inhibited their responses and decreased by $50 \mathrm{msec}$ if they could not. Since it is harder to inhibit with longer delays, this dynamic tracking method ensured that participants successfully inhibited $50 \%$ of stop trials. SSRT could then be computed according to the race model of stopping (Logan \& Cowan, 1984), using the formula SSRT $=$ median go RT - SSD.

Knowledge questionnaire. The knowledge questionnaire was adapted from previous SRT studies (Fletcher et al., 2005; Willingham et al., 1989). Open-ended questions probed participants about the extent of their knowledge of the sequence. For example, they were asked "Did you notice any pattern or repeating sequence?" and "Did you ever feel that you could predict the next position?" After each question, an example was requested from participants, who were urged to write down any sequence that they noticed using free recall.

\section{Procedure}

Participants were trained on the above tasks for three 1-h sessions within 1 week. After Session 3, they were given the knowledge questionnaire. Seven participants were excluded from analyses because their calculated SSRTs were negative; this reflected deliberate slowing of go responses, contrary to task instructions. Consequently, all analyses were conducted with 40 participants (mean age, 21.3 years), 24 women and 16 men.

\section{Data Analysis}

The data were analyzed using a repeated measures ANOVA in SPSS (SPSS, Chicago, IL) with the Huyhn-Feldt correction for nonsphericity. Only correct responses were included in RT analyses.

\section{RESULTS}

\section{Knowledge Questionnaire}

Participants were separated into two groups on the basis of their knowledge questionnaire responses. Explicit knowledge was operationalized as correctly identifying, in order, four or more locations during free recall. Participants in the implicit knowledge group could not correctly identify more than two sequential target locations of the sequence; $33 \%$ of participants (13 out of 40 ) had explicit knowledge. The average number of consecutive sequence locations they reported was 5.2.

\section{Sequence Effect}

To determine whether participants learned the sequence, single-task blocks were examined in an ANOVA with sequence knowledge (explicit/implicit) as the betweengroups variable, and session (1-3), block (1-12), and condition (sequenced/random) as the within-groups repeated variables.
First, median RT was explored. RT decreased across training sessions $[F(2,76)=42.20, p<.001 ; 389.7$ $(S E=7.13), 368.7(S E=6.8)$, and $359.5(S E=$ 6.9) msec, respectively]. RT also decreased within each block $[F(11,418)=3.09, p<.01]$. Additionally, participants were faster during sequenced than during random blocks $[F(1,38)=119.07, p<.001$; median RT 361.1 $(S E=7.2)$ vs. $384.2(S E=6.3) \mathrm{msec}$ for sequenced and random, respectively]. Lastly, participants with explicit knowledge tended to be marginally faster than those with implicit knowledge $[F(1,38)=3.64, p=.06$; median RT $359.9(S E=11.0)$ vs. $385.3(S E=7.6)$ msec for explicit and implicit, respectively].

There were also significant interactions. First, there was a greater discrepancy between sequenced and random blocks in the explicit knowledge group than in the implicit knowledge group [condition $\times$ knowledge interaction: $F(1,38)=11.22, p<.01]$. Second, throughout training RT decreased more during sequenced than during random blocks [session $\times$ condition interaction: $[F(2,76)=14.73, p<.001]$. Lastly, it was observed that the session $\times$ condition interaction tended to be greater for participants with explicit knowledge than for those with implicit knowledge, as seen by the marginally significant session $\times$ condition $\times$ knowledge interaction $[F(2,76)=$ $3.14, p=.06]$. To further explore that interaction, we conducted a session $\times$ condition ANOVA separately for the explicit and implicit knowledge groups. Indeed, the session $\times$ condition interaction was significant for both explicit $[F(2,24)=8.94, p<.001]$ and implicit $[F(2,52)=$ $4.69, p=.02]$ knowledge groups, albeit greater in magnitude for the explicit group (see Figure 2A).

Next, accuracy data were examined. All participants were highly accurate (mean accuracy 96\% [SE $=0.4 \%]$, ranging from $95.9 \%$ to $96.3 \%$ for all groups/sessions/conditions). Potentially due to a ceiling effect, there was no main effect of session. Participants were more accurate on sequenced than on random blocks $[F(1,38)=13.74$, $p=.001$; accuracy $96.5 \%(S E=0.4)$ vs. $95.7 \%(S E=$ 0.4 ) for sequenced and random blocks, respectively]. There was also a greater discrepancy in accuracy between sequenced and random blocks throughout training [session $\times$ condition interaction: $F(2,76)=3.23, p=.045]$. Lastly, participants became less accurate throughout each block, likely due to fatigue $[F(11,165)=2.51, p=.02]$; see Figure 2B.

\section{Dual-Task Effect}

As an index of automaticity, the effect of the secondary task was measured at six points throughout training (at the beginning and end of each session). It was calculated separately for sequenced and random blocks. RT data of the two adjacent dual-task blocks for each condition were averaged together (e.g., Blocks 7 and 9 or 8 and 10) and compared with the average of the surrounding single-task blocks (e.g., Blocks 5 and 11 or 6 and 12), with the dual-task effect defined as the difference between those averages.

Accuracy on the secondary task was measured by computing the percent distance of the participant's reported 

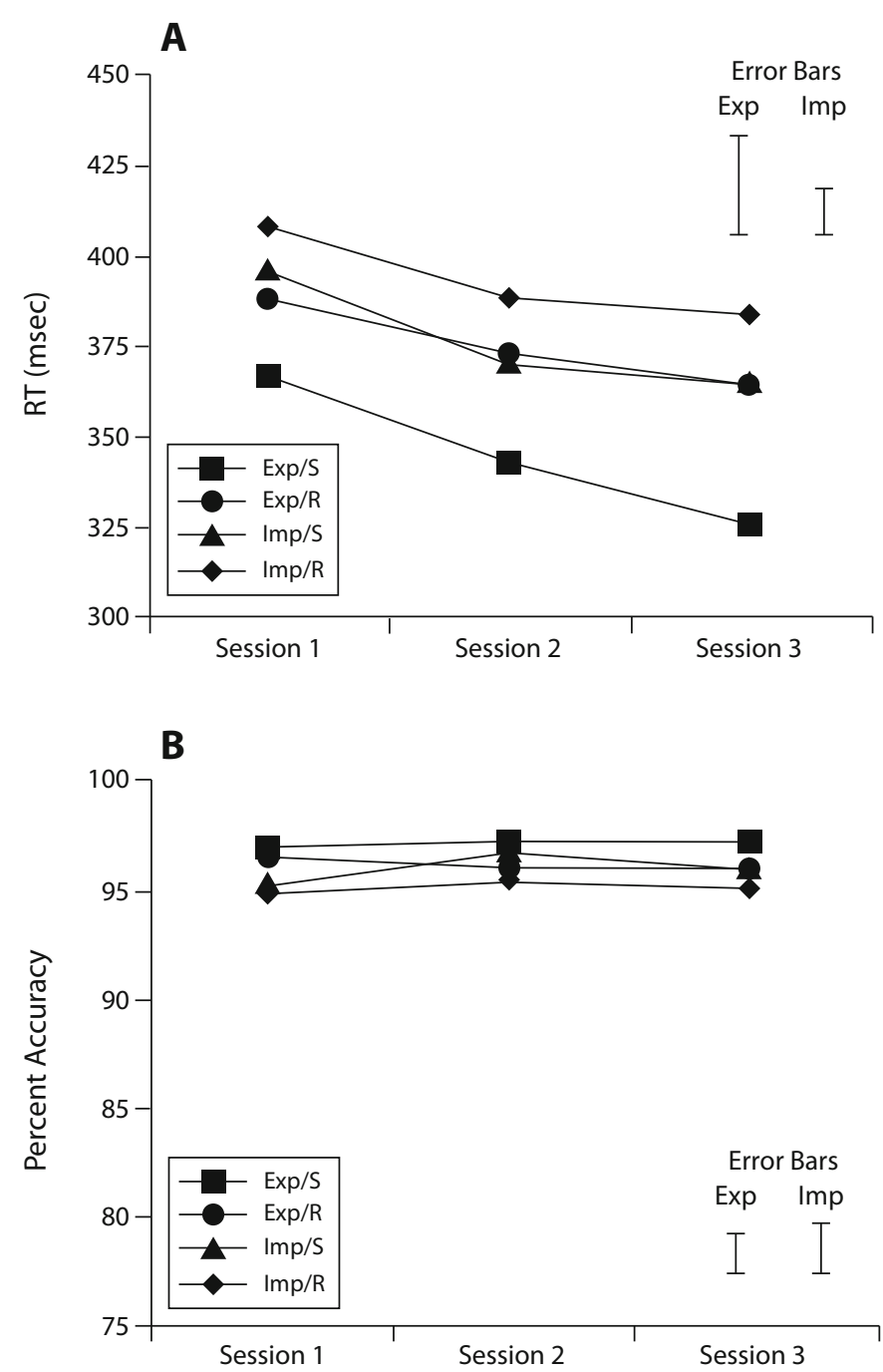

Figure 2. SRT sequence effect on single-task blocks for median reaction time (RT) (A) and percent accuracy (B). Error bars are presented on the basis of within-subjects standard error. Exp, explicit knowledge; Imp, implicit knowledge; S, sequenced blocks; R, random blocks.

number of targets from the actual number of targets $(100 \times$ |participant response - correct answer $/$ correct answer). The median error rate was low, ranging from $0.86 \%$ to $4.78 \%$ during the six time points in which the secondary task occurred.

Analyses compared the dual-task effect at the beginning of Session 1 to that at the end of Session 3, in order to determine the degree of change in automaticity from the beginning to the end of training. Dual-task blocks were examined in an ANOVA with sequence knowledge (explicit/implicit) as the between-groups variable and session $(1 / 3)$ and condition (sequenced/random) as the withingroups repeated variables. The dual-task effect decreased with training [main effect of session: $F(1,38)=20.04$; $p<.001$ ], implying that the SRT became more automatic. There were no other significant main effects or interactions (all $p \mathrm{~s}>.13$; see Figure 3 ).
The dual-task effect at the end of training was significantly different from zero for the entire group of participants $(p<.001$ for both sequenced [27.8 msec] and random [30.3 msec] conditions), indicating that the SRT did not become fully automatic by the end of training. To examine differences within the group, we used a median split of dual-task effect in Session 3 to separate all participants into two groups. The group below the median showed no significant dual-task effects (means of $-3.5 \mathrm{msec}$ for sequenced and $0.2 \mathrm{msec}$ for random blocks; both $p$ s comparing the dual-task effects to zero $>.4$ ), indicating that the dual-task effect was eliminated for these participants in both sequenced and random conditions. Given the lack of any dual-task effect, we termed this group the "fully automatic" group. The "less automatic" group was defined as those whose dual-task effects were above the median (means of $59.1 \mathrm{msec}$ for sequenced and $60.5 \mathrm{msec}$ for ran- 


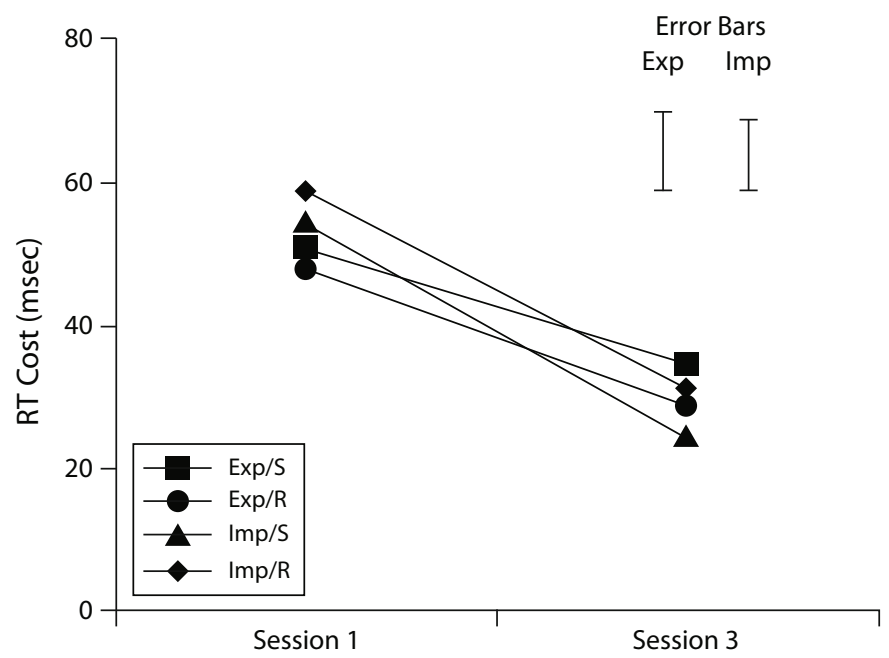

Figure 3. Dual-task effect on dual-task blocks for reaction time (RT). Dual-task effect is defined as the RT cost on the SRT during dual-task blocks in comparison with adjacent single-task blocks. Error bars are presented on the basis of within-subjects standard error. Exp, explicit knowledge; Imp, implicit knowledge; $S$, sequenced blocks; R, random blocks.

dom blocks; both $p$ s comparing the dual-task effects to zero $<.001)$. Although the SRT did become significantly more automatized for the less automatic group, there was a remaining dual-task cost.

SRT accuracy did not differ between single- and dualtask blocks. The average difference in accuracy for all groups, time points, and conditions between the dual task and single task was less than $1 \%$. The lack of significant effects (all $p \mathrm{~s}>.17$ ) may reflect a ceiling effect.

\section{Stop-Signal RT}

In order to validly use the race model of stopping (Logan \& Cowan, 1984) to calculate SSRT, it is necessary that participants successfully inhibit their responses on $50 \%$ of stop trials. To allow time for the SSD staircases to converge and to therefore ensure $50 \%$ inhibition, we used the data from the second half of each time point in which we measured SSRT to calculate our SSRT estimates. Averaging over the second half of all stop-signal blocks, participants inhibited their responses on $50.3 \%$ of stop trials (range, $48.9 \%-51.5 \%$ ), a value not significantly different from $50(p>.2)$.

In order to test the hypothesis that the level of automaticity on the SRT does not impair response inhibition, analyses compared SSRT at the beginning of Session 1 with SSRT at the end of Session 3. Stop-signal blocks were examined in an ANOVA with sequence knowledge (explicit/implicit) as the between-groups variable and session (1/3) and condition (sequenced/random) as the within-groups repeated variables.

Crucially, the level of automaticity of the SRT did not influence participants' ability to withhold responses on the SRT, as reflected in the lack of significant effects in the analysis of SSRT (all $p s>.2$; see Figure 4A). In further support of that conclusion, comparison of the fully automatic and less automatic groups showed no difference in change in SSRT from Session 1 to Session 3 between the two groups [one-tailed $t$ tests: $t(38)=0.55, p=.59$ for sequenced and $t(38)=0.92, p=.36$ for random; see Table 1]. With 40 participants, this analysis had $80 \%$ power to detect a large effect size (Cohen's $d=0.8$ ), giving confidence that if there is an effect of automaticity on response inhibition, it is not substantial. Moreover, there were no significant effects with SSRT for the fully automatic group alone (all $p s>.2$ ). Further analyses demonstrated that the decrease in dual-task effect was not correlated with the change in SSRT from Session 1 to Session 3 in either sequenced $(p=.8)$ or random $(p=.3)$ blocks, again suggesting that the level of automaticity of the SRT did not have an effect on one's ability to inhibit one's responses.

Participants became less accurate across sessions on the SRT on stop-signal blocks [main effect of session: $F(1,38)=5.41, p=.03]$. Further exploration revealed that this effect of session was likely due to fatigue. When including all time points of stop-signal blocks in a 3 (session: $1-3) \times 2$ (time point: early/late) $\times 2$ (condition: sequenced/random) ANOVA, it was revealed that within each session participants became less accurate on the

Table 1

Summary Statistics for Comparison of Change (in Milliseconds) in SSRT, with Standard Errors (SEs), From Session 1 to Session 3 for the Fully Automatic and Less Automatic Groups in Sequenced and Random Conditions

\begin{tabular}{crrrrr}
\hline & \multicolumn{2}{c}{$\begin{array}{c}\text { Fully } \\
\text { Automatic }\end{array}$} & & \multicolumn{2}{c}{$\begin{array}{c}\text { Less } \\
\text { Automatic }\end{array}$} \\
\cline { 2 - 3 } \cline { 5 - 6 } Condition & $M$ & $S E$ & & $M$ & $S E$ \\
\hline Sequenced & 2.8 & 19.6 & & -11.2 & 16.3 \\
Random & -0.5 & 17.6 & & -23.9 & 18.3 \\
\hline
\end{tabular}



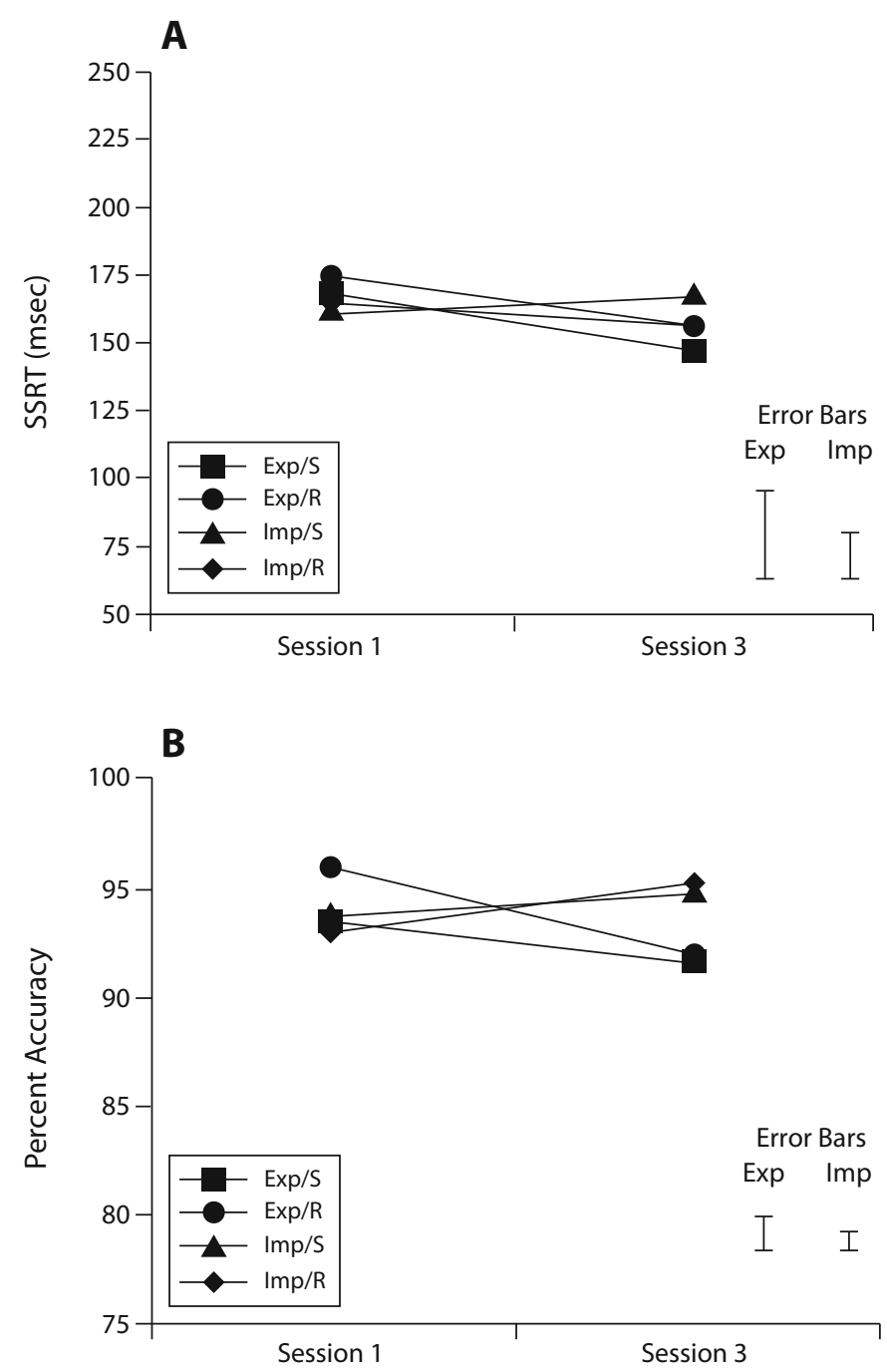

Figure 4. Stop-signal reaction time (A) and SRT accuracy (B) on stopsignal blocks. Error bars are presented on the basis of within-subjects standard error. Exp, explicit knowledge; Imp, implicit knowledge; S, sequenced blocks; $R$, random blocks.

SRT on stop-signal blocks $[F(1,38)=15.65, p<.001]$. There was no main effect of session $(p=.8)$, indicating that across sessions, participants did not become less accurate on the SRT during stop-signal blocks. In Session 1, participants with explicit knowledge were more accurate than those with implicit knowledge, but the opposite was true for Session 3 [session $\times$ knowledge interaction: $F(1,38)=5.53, p=.02]$. There were no other significant main effects or interactions (all $p \mathrm{~s}>.4$ ); see Figure 4B.

\section{DISCUSSION}

The present results demonstrate that, although training on the SRT was associated with the development of automaticity as measured by a dual-task manipulation, there was no change in concomitant ability to inhibit motor responses on the SRT. These results contradict the proposal that automaticity is associated with a loss of control or development of ballistic movements. Our results confirm Logan's (1982) previous result that expert typists were not impaired in stopping ongoing typing performance, even though their behavior was highly automatized. These data converge to suggest that automaticity in certain motor tasks is unrelated to the ability to exert some forms of control over those movements.

Three possible modes through which extensive practice could reduce dual-task interference have been proposed in the literature exploring effects of central executive bottlenecks in processing and response selection: integration of the two tasks into one complex task, shortened processing times of the practiced task, or automation of the practiced task (Ruthruff, Van Selst, Johnston, \& Remington, 2006). The first two would imply that the SRT is still somewhat controlled, as operationalized by continued reliance on the central executive and a persistent bottleneck, but-either 
by combining the SRT and tone-counting tasks into a well-integrated "supertask," or by shortening the duration of the processing stage that is dependent on the central executive - dual-task interference may be reduced. Only the third option would eliminate the need for a central executive and therefore indicate true automaticity. Ruthruff et al. noted individual differences in their participants, in that most showed evidence of "stage shortening," such that, with practice, they were able to proceed with the primary task more quickly. Therefore, central processing of the second task could occur sooner and reduce dual-task interference. In some participants, however, it appeared that one of the tasks was automatized and no longer relied on the central executive, as evidenced by reduced dualtask interference without a proportional decrease in RT (in order for stage shortening to occur, the task would need to be processed more quickly, and RTs would therefore decrease). Additionally, practice on either the first or second task reduced dual-task interference. With stage shortening only, practice on the first task should reduce dualtask interference, since processing of the second task may not begin until processing of the first task is completed, whereas dual-task interference will decrease if either of the tasks is automatized.

It appears that although automaticity (in the sense defined by Ruthruff et al., 2006) is possible, it is rare and occurs only under certain circumstances (Lien, Ruthruff, \& Johnston, 2006). However, the present task may be an example of such a case. First, the SRT is "ideomotor compatible," meaning that the stimuli and responses are intuitively compatible. Second, because the tone-counting task did not require a response while performing the SRT, there are no competing responses. Third, post hoc analyses demonstrated that RTs and accuracy for each session (sequenced and random separately) were not significantly different for the groups that differed in their sensitivity to the secondary task (all $p s>$.2) (Lien et al., 2006; Ruthruff et al., 2006). Therefore, it appears that stage shortening, which results in decreased RTs, may not fully explain the results. A more conclusive determination of this question would call for a study in which the secondary task required a response on every trial, so its interference could be measured on a trial-by-trial basis.

It is further possible that the reduction in dual-task effect reflected an increased ability to switch between the two simultaneous tasks (SRT and letter-counting), as opposed to a decreased amount of resources being required to perform the SRT due to increased automaticity (Pashler, 1998). However, there was very little practice under dual-task conditions (10 blocks out of 120 total), meaning that there was not much opportunity to practice task switching. Additionally, there is ample evidence for reduced dual-task interference in highly practiced stimulusresponse tasks, and much of the data used to support the task-switching theory was with visual search tasks, not stimulus-response tasks (Pashler, 1998). Therefore, it is likely that the reduced dual-task interference did in fact reflect increased automaticity of the SRT.
It is also feasible that the decrease in dual-task effect could have been due to a shift in relative attention from the secondary task to the SRT over time. However, we found that participants were highly accurate on the secondary task throughout all sessions. In fact, they became more accurate over time, as noted in a paired $t$ test comparing secondary task accuracy at the beginning of Session 1 to that at the end of Session $3[t(39)=5.75, p<.001]$. Therefore, participants improved on both the SRT and the secondary task over time, suggesting that reductions in the dual-task effect cannot be attributed to a shift in attention toward the SRT.

It is worth noting that although the SRT did not become fully automatic after $3 \mathrm{~h}$ of training for all participants, as operationalized by an absence of a dual-task effect, there was a subset of participants for whom the SRT did become fully automatic. For these participants, the dual-task effect was eliminated in both sequenced and random conditions. Basic features of both conditions, such as the tuning of compatible stimulus-response mappings with practice as well as with general practice effects may make both conditions become automatic, even though the sequenced condition seems to be easier for participants overall, as operationalized by performance being faster and more accurate on sequenced than on random blocks (Poldrack et al., 2005). There was no difference in response inhibition ability between the fully automatic and the less automatic groups. A linear regression analysis with dual task effect at Session 3 as the dependent variable and change in SSRT from Session 1 to Session 3 as the independent variable indicated that level of automaticity as indexed by final dual task effect was not a significant predictor of SSRT in either sequenced $\left(R^{2}=.016\right)$ or random $\left(R^{2}=\right.$ .013 ) blocks (both $p s>.4$ ).

Our results suggest that it is possible to exert motor control over automatized behavior in tasks such as the SRT (or typing; see Logan, 1982). Some evidence exists that other forms of control, such as attentional control, may be more difficult to suppress with automatic behavior. For example, Treisman, Vieira, and Hayes (1992) found that training participants on a visual search task until it became automatized resulted in irrelevant stimulus dimensions either benefiting (if they were consistent) or impairing (if they were inconsistent) performance on the task, as assessed by speed. They concluded that attention to irrelevant stimulus dimensions could not be controlled in an automatic visual search task. Similarly, the Stroop effect (Stroop, 1935) implies that, due to the highly automatic process of reading, attention to irrelevant dimensions of a stimulus (the color word itself, such as green) cannot be suppressed, even when participants are asked to name the ink color in which the word is printed. This effect never disappears, but it can be reduced with practice, implying that the automatic process of reading can be modulated and is not purely uncontrollable (Pashler, 1998).

Furthermore, it is important to draw a distinction between autonomous and automatic tasks. Autonomous tasks may be defined as both beginning and ending with- 
out intention (Zbrodoff \& Logan, 1986). Tasks that are automatic may or may not be completely autonomous, assuming that autonomy is a continuous dimension. For example, reading the word in the Stroop interference task is commonly noted to be automatic, in that one cannot fully inhibit reading the word when instructed to name the ink color, thus slowing down naming responses when the stimulus is incongruent in the two dimensions. However, it is possible to inhibit the reading response in order to make the correct naming response; the Stroop effect, therefore, does not run to completion autonomously. It may be automatic without being completely autonomous, as noted by the ability of participants to interrupt the completion of the task with a signal to stop. Further support for this lies in the analysis that the degree of automaticity on the SRT, as indexed by change in dual task effect from Session 1 to Session 3, is unrelated to the change in the degree of control one has over the SRT, as indexed by SSRT on the stopping task.

In summary, training on the SRT leads to automaticity as defined by the commonly accepted criterion of elimination of dual-task interference, while the ability to control behavior remains unchanged. Although questions remain regarding the underlying mechanisms for this reduction in dual-task interference, the results converge with the results of Logan's (1982) study of expert typists to suggest that inhibitory control is unaffected by the development of skill.

\section{AUTHOR NOTE}

Parts of this research were presented at the 35th Annual Meeting of the Society for Neuroscience in Washington, DC, in 2005. This research was sponsored by the McDonnell Foundation 21st Century Science Program (to R.A.P.). The authors thank Gordon Logan, Hal Pashler, and an anonymous reviewer for helpful comments on an earlier version. Correspondence concerning this article should be addressed to J. R. Cohen, Department of Psychology, University of California, Los Angeles, 1285 Franz Hall, Box 951563, Los Angeles, CA 90095-1563 (e-mail: jrcohen@psych.ucla.edu).

\section{REFERENCES}

Aizenstein, H. J., Stenger, V. A., Cochran, J., Clark, K., Johnson, M., Nebes, R. D., \& Carter, C. S. (2004). Regional brain activation during concurrent implicit and explicit sequence learning. Cerebral Cortex, 14, 199-208.

Band, G. P. H., van der Molen, M. W., \& Logan, G. D. (2003). Horse-race model simulations of the stop-signal procedure. Acta Psychologica, 112, 105-142.

Brainard, D. H. (1997). The Psychophysics Toolbox. Spatial Vision, 10, 433-436.

Fletcher, P. C., Zafiris, O., Frith, C. D., Honey, R. A. E., Corlett, P. R., Zilles, K., \& FinK, G. R. (2005). On the benefits of not trying: Brain activity and connectivity reflecting the interactions of explicit and implicit sequence learning. Cerebral Cortex, 15, 1002-1015.

Hazeltine, E., Grafton, S. T., \& Ivry, R. (1997). Attention and stimulus characteristics determine the locus of motor-sequence encoding: A PET study. Brain, 120, 123-140.

Lien, M., Ruthruff, E., \& Johnston, J. C. (2006). Attentional limita- tions in doing two tasks at once: The search for exceptions. Current Directions in Psychological Science, 15, 89-93.

Lijffijt, M., Kenemans, J. L., Verbaten, M. N., \& Van Engeland, H. (2005). A meta-analytic review of stopping performance in attentiondeficit/hyperactivity disorder: Deficient inhibitory motor control? Journal of Abnormal Psychology, 114, 216-222.

LogAN, G. D. (1979). On the use of a concurrent memory load to measure attention and automaticity. Journal of Experimental Psychology: Human Perception \& Performance, 5, 189-207.

LoGAN, G. D. (1982). On the ability to inhibit complex movements: A stop-signal study of typewriting. Journal of Experimental Psychology: Human Perception \& Performance, 8, 778-792.

LoGAN, G. D. (1983). Time, information, and the various spans in typewriting. In W. E. Cooper (Ed.), Cognitive aspects of skilled typewriting (pp. 197-224). New York: Springer.

Logan, G. D. (1994). On the ability to inhibit thought and action: A users' guide to the stop signal paradigm. In D. Dagenbach \& T. H. Carr (Eds.), Inhibitory processes in attention, memory and language (pp. 189-240). San Diego: Academic Press.

Logan, G. D., \& Cowan, W. B. (1984). On the ability to inhibit thought and action: A theory of an act of control. Psychological Review, 91, 295-327.

Nissen, M. J., \& Bullemer, P. (1987). Attentional requirements of learning: Evidence from performance measures. Cognitive Psychology, 19, 1-32.

PaShler, H. E. (1998). The psychology of attention. Cambridge, MA: MIT Press.

Perruchet, P., \& Amorim, M.-A. (1992). Conscious knowledge and changes in performance in sequence learning: Evidence against dissociation. Journal of Experimental Psychology: Learning, Memory, \& Cognition, 18, 785-800.

Poldrack, R. A., SAbb, F. W., Foerde, K., Tom, S. M., Asarnow, R. F., BookHEIMER, S. Y., \& KNOwLton, B. J. (2005). The neural correlates of motor skill automaticity. Journal of Neuroscience, 25, 5356-5364.

Posner, M. I., \& SNyder, C. R. R. (1975). Attention and cognitive control. In R. L. Solso (Ed.), Information processing and cognition: The Loyola symposium (pp. 55-85). Hillsdale, NJ: Erlbaum.

Ruthruff, E., Van Selst, M., Johnston, J. C., \& Remington, R. (2006). How does practice reduce dual-task interference: Integration, automatization, or just stage-shortening? Psychological Research, 70, 125-142.

SAlthouse, T. A. (1984). Effects of age and skill in typing. Journal of Experimental Psychology: General, 113, 345-371.

Schneider, W., \& Shiffrin, R. M. (1977). Controlled and automatic human information processing: I. Detection, search, and attention. Psychological Review, 84, 1-66.

Shiffrin, R. M., \& SCHNEIDER, W. (1977). Controlled and automatic human information processing: II. Perceptual learning, automatic attending, and a general theory. Psychological Review, 84, 127-190.

Stroop, J. R. (1935). Studies of interference in serial verbal reactions. Journal of Experimental Psychology, 18, 643-662.

Treisman, A., Vieira, A., \& Hayes, A. (1992). Automaticity and preattentive processing. American Journal of Psychology, 105, 341-362.

Willingham, D. B., Nissen, M. J., \& Bullemer, P. (1989). On the development of procedural knowledge. Journal of Experimental Psychology: Learning, Memory, \& Cognition, 15, 1047-1060.

Willingham, D. B., Salidis, J., \& Gabrieli, J. D. E. (2002). Direct comparison of neural systems mediating conscious and unconscious skill learning. Journal of Neurophysiology, 88, 1451-1460.

Zbrodoff, N. J., \& LoGan, G. D. (1986). On the autonomy of mental processes: A case study of arithmetic. Journal of Experimental Psychology: General, 115, 118-130.

(Manuscript received November 21, 2006; revision accepted for publication July 12, 2007.) 